OPEN ACCESS

Edited by:

In-Kyu Park,

Chonnam National University,

South Korea

Reviewed by:

Kai Xiao,

Sichuan University, China

Renjith P. Johnson,

Yenepoya University, India

${ }^{*}$ Correspondence:

Paul Apka

paulapka275@gmail.com

Specialty section:

This article was submitted to

Nanobiotechnology,

a section of the journal

Frontiers in Bioengineering and

Biotechnology

Received: 16 December 2020

Accepted: 27 August 2021

Published: 15 December 2021

Citation:

Venkataraman S, Apka P, Shoeb E, Badar U and Hefferon K (2021) Plant Virus Nanoparticles for Anticancer Therapy.

Front. Bioeng. Biotechnol. 9:642794.

doi: 10.3389/fbioe.2021.642794

\section{Plant Virus Nanoparticles for Anti-cancer Therapy}

\author{
Srividhya Venkataraman ${ }^{1}$, Paul Apka ${ }^{2,3 *}$, Erum Shoeb ${ }^{1,4}$, Uzma Badar $^{1,4}$ and \\ Kathleen Hefferon ${ }^{1}$
}

${ }^{1}$ Department of Cell and Systems Biology, University of Toronto, Toronto, ON, Canada, ${ }^{2}$ Theranostics and Drug Discovery Research Group, Faculty of Pharmaceutical Sciences, University of Nigeria, Nsukka, Nigeria, ${ }^{3}$ Department of Pharmaceutics, Faculty of Pharmaceutical Sciences, University of Nigeria, Nsukka, Nigeria, ${ }^{4}$ Department of Genetics, University of Karachi, Karachi, Pakistan

Plant virus nanoparticles (VNPs) are inexpensive to produce, safe, biodegradable and efficacious as treatments. The applications of $r$ plant virus nanoparticles range from epitope carriers for vaccines to agents in cancer immunotherapy. Both VNPs and viruslike particles (VLPS) are highly immunogenic and are readily phagocytosed by antigen presenting cells (APCs), which in turn elicit antigen processing and display of pathogenic epitopes on their surfaces. Since the VLPs are composed of multiple copies of their respective capsid proteins, they present repetitive multivalent scaffolds which aid in antigen presentation. Therefore, the VLPs prove to be highly suitable platforms for delivery and presentation of antigenic epitopes, resulting in induction of more robust immune response compared to those of their soluble counterparts. Since the tumor microenvironment poses the challenge of self-antigen tolerance, VLPS are preferrable platforms for delivery and display of self-antigens as well as otherwise weakly immunogenic antigens. These properties, in addition to their diminutive size, enable the VLPS to deliver vaccines to the draining lymph nodes in addition to promoting APC interactions. Furthermore, many plant viral VLPs possess inherent adjuvant properties dispensing with the requirement of additional adjuvants to stimulate immune activity. Some of the highly immunogenic VLPS elicit innate immune activity, which in turn instigate adaptive immunity in tumor microenvironments. Plant viral VLPs are nontoxic, inherently stable, and capable of being mass-produced as well as being modified with antigens and drugs, therefore providing an attractive option for eliciting anti-tumor immunity. The following review explores the use of plant viruses as epitope carrying nanoparticles and as a novel tools in cancer immunotherapy.

Keywords: nanoparticles, plant virus-like particles, therapeutics, imaging, cancer 2

\section{INTRODUCTION}

Nanomedicine is an emerging area of multidisciplinary research that has already shown promise of transforming into a disruptive innovative development (Farokhzad et al., 2008). Already, there are dozens of products in clinical trials and even some on the shelf in some pharmacies across the world though users are relatively few because of the rather prohibitive price tags of these innovative products (Park, 2019). It is pertinent to state that while a lot has been proposed in terms of the 
anticipated efficacy of nanomedicines, opinions tend to vary when it comes to the stage of a critical cost-benefit- analysis for the availability of nanomedicines for use in the treatment of cancer and other ailments (FDA Drug Reports, 2017).

Nanomedicines includes a wide array of nanomaterials with particle size ranging from $1 \mathrm{~nm}$ to more than $400 \mathrm{~nm}$ and are a remarkably diverse group of materials (Zhang et al., 2008; Zhou et al., 2012). They may be made up of entirely of a metal as in the case of Gold and Silver nanoparticles (Paviolo and Stoddart, 2017), or a combination of liquids or a ternary system composed of an assortment of several compatible materials giving rise in most cases to a multifunctional entity often possessing stimuli responsive attributes enabling it to respond to minute changes in factors such as $\mathrm{pH}$ and temperature variations (Moreira et al., 2016). Additionally, nanoparticles can be prepared with simple polymeric materials such as cellulose and chitosan (Steinmetz and Manchester, 2009).

Immunotherapy in cancer treatment simply refers to a strategy with the objective of galvanizing the immune system of the patient to resist the implanting of cancerous cells. There are several approaches to achieve the desired end. One approach involves the use of drugs known as "Immune checkpoint inhibitors, to block immune checkpoints (Byun et al., 2017). The checkpoints are a typical part of the immune system and serve to modulate the immune response so that it does not come as too strong. The net effect of this treatment modality is that the blocking of these checkpoints makes it possible for the immune cells to respond more strongly to cancer.

Plant virus-based nanoparticles (VNPs) have been explored as a unique class of nanocarriers for biomedical applications (Pitek et al., 2016). In addition to their ease of production and quality control maintenance, plant virus VNPs offer a logical alternative to synthetic nanoparticles as they are inexpensive to produce, nontoxic and biodegradable (Rybickie, 2020). Plant virus nanoparticles have been further improved for their performance in terms of stimuli-responsivity (Brun, Gomez, and Suh 2017).

Plant virus nanoparticles tend to be either rod shaped, such as Tobacco mosaic virus (TMV) and Potato virus X (PVX), or icosahedral shaped, such as Cowpea mosaic virus (CPMV). Different shaped viruses respond differently as nanoparticles in vivo. Tobacco mosaic virus can assemble into VLPs without requiring its RNA genome carry a drug payload on the surface or to a limited extent, within the inner channel of the nanoparticle. Potato virus X, cannot self-assemble in the absence of its RNA genome, and thus can only carry a payload on the outer surface. Cowpea mosaic virus can be made to self-assemble into empty virus like particles in the absence of its RNA genome and can thus carry a payload both inside and outside of its protein shell (Sainsbury et al., 2010). In this review, we provide a series of examples to discuss how plant virus architecture contributes to their applications in cancer diagnostics and therapy (Wen et al., 2015a; Wen et al., 2015b). We discuss the architecture of plant viruses, how they came to be used as nanoparticles in various medical applications, and how they may be employed in the future as novel cancer immunotherapies (Shahgolzari et al., 2021).

\section{Architecture of Plant Virus Nanoparticles}

Viruses are composed of outer protein shells which encapsulate the genomic material. The multiple copies of coat proteins that form the virus outer shell of viruses are collectively known as the capsid (Liu et al., 2016). Primarily, the capsid occurs in different shapes and sizes and is meant to protect the genomic material to keep viruses safe under extreme environments (Pokorski and Steinmetz 2011). The immense diversity with respect to the shape and size of plant viruses enables them to be tailored for specific applications. The structural integrity of viruses remains intact even when surface properties have been altered through chemical and genetic modification; this allows control over targeting ligands, drugs and contrast agents for imaging (Rong et al., 2011). Various chemical and genetic approaches are reported to control the virus surface properties without affecting structural integrity, and allow control on the attachment sites of drug molecules or contrast agents on the virus surface (Rong et al., 2011). Plant-virus capsid pores are also reported to be employed to encapsulate small therapeutic molecules (Zeng et al., 2013).

Plant viruses have been used as virus like particles (VLPs) and virus nanoparticles (VNPs) as epitope display systems for vaccine production. VLPs are a subset of the VNPs but lack any nucleic acid genome, thus making them non-infectious. VNPs and VLPs based on plant viruses are both non-pathogenic to humans and biodegradable (Steinmetz, 2010). VNPs and VLPs are advantageous due to their ability to be generated quickly while serving as highly versatile molecular scaffolds (Young et al., 2008; Steinmetz and Evans, 2007]. Examples of plant viruses utilized as VLPs include Cowpea mosaic virus (CPMV) and Tobacco mosaic virus (TMV). An example of a plant virus utilized as a VNP is Potato virus X (PVX). These are listed in Figure 1.

Tobacco mosaic virus (TMV) is the most well-studied plant virus and was initially characterized in the 19th century. TMV can be easily produced and purified in bulk amounts and can be manipulated genetically due to its relatively simple particle structure and genome organization. The rod-shaped virus particle measures $300 \mathrm{~nm}$ in length and $18 \mathrm{~nm}$ in diameter and contains a $6.7 \mathrm{~kb}$ viral RNA genome that is encapsulated by 2,130 identical copies of the capsid protein assembled in a helical arrangement. At neutral $\mathrm{pH}$ and in the absence of RNA, the $\mathrm{CP}$ assembles itself into an $18 \mathrm{~nm}$ double disk, a 20S aggregate or nano-ring containing two layers of $17 \mathrm{CP}$ molecules which can serve as a nanoscale scaffold.

Potato virus $\mathrm{X}$ (PVX) is a member of the family Alphaflexiviridae, genus Potexvirus, an important plant pathogen of the family Solanaceae, and specifically infects potato, tomato and tobacco (Adams et al., 2004) (Massumi et al., 2014). It has a 6.4-kb positive-stranded RNA genome (Park et al., 2008). Multiple copies of CP assemble around the genomic RNA to form the capsid. PVX can carry large payloads due to its flexible and filamentous structure, making it possible to use for pharmaceutical and imaging applications (Roder, Dickmeis, and Commandeur 2019).

The PVX particle is $515 \times 14.5 \mathrm{~nm}$ in dimension and comprised of 1,270 subunits of CP, (Parker et al., 2002). The C-terminus of each CP subunit is located internally and the 
A

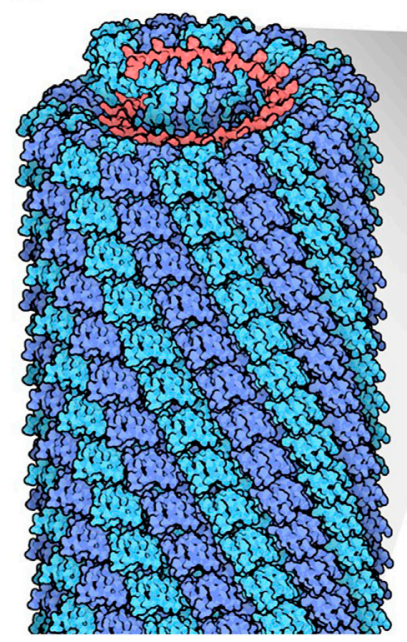

B

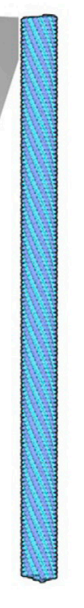

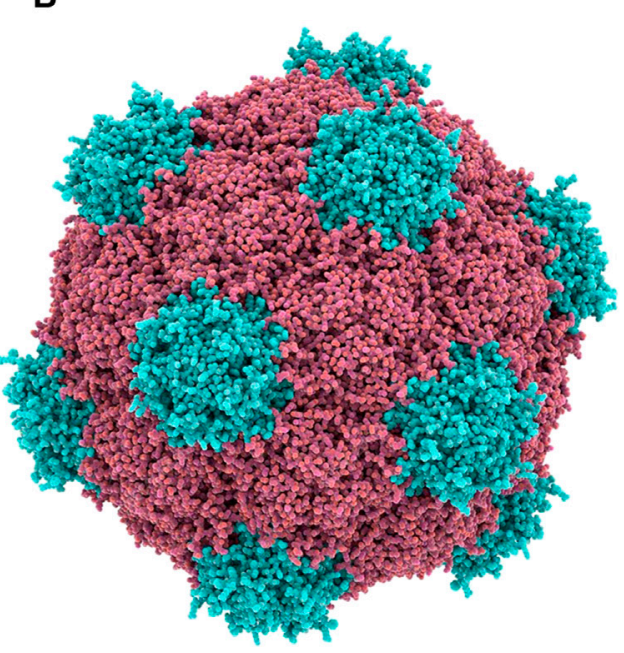

c

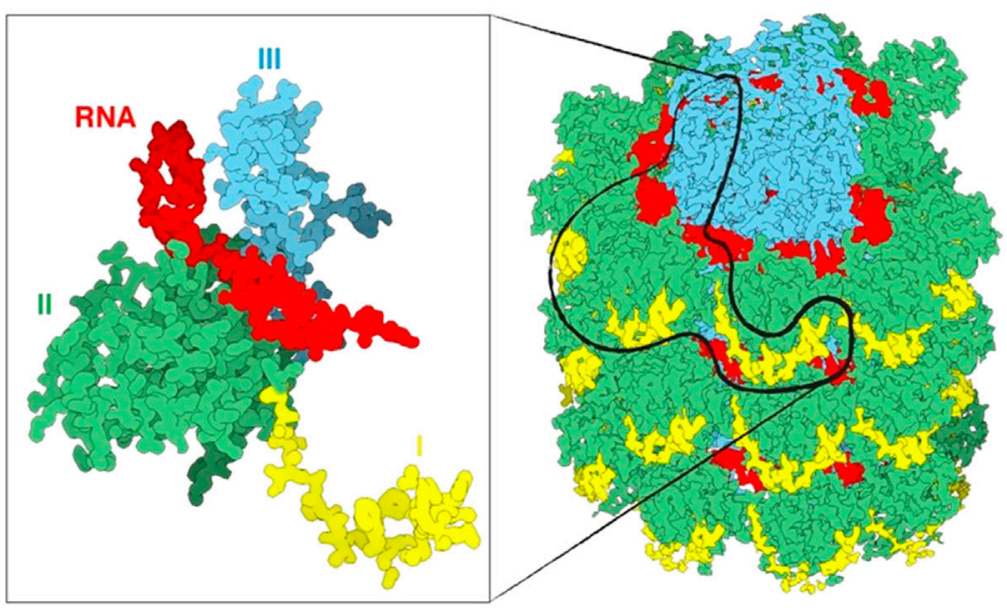

FIGURE 1 | (A) Tobacco mosaic virus structure, RNA is in red, protein subunits in blue Source: https://pdb101.rcsb.org/motm/109. (B) Cowpea mosaic virus structure, Protein subunits in red and blue Source: fineartamerica. com. (C) An overview of a portion of the PVX virus (right). The three domains of the protein are shin in yellow, green and cyan, the RNA in red. The magnification on the left displays only one single CP with a fragment of RNA.

N-terminus projected externally to the assembled particle, which provides a suitable site for modification (Nemykh et al., 2008). Unlike other reported viruses, the assembly of PVX CP subunits into filamentous VLP, in vivo or in vitro, is not possible in the absence of genomic RNA. This reflects the unique connection between virus RNA and CP (Kwon et al., 2005).

The plant pathogen Cowpea mosaic virus (CPMV) belongs to the Comovirus genus. CPMV is an icosahedral shaped virus with a diameter of approximately $27 \mathrm{~nm}$. It is composed of RNA-1 and RNA-2 of 6 and $3.5 \mathrm{~kb}$, respectively packed in 60 copies each of Large and Small coat protein (Singh et al., 2007). CPMV is one of the most developed VNPs for biomedical and nanotechnology applications due to its ability to target specific tissues and act as an efficient drug delivery system. It is also reported to be welladapted for the attachment of a variety of molecules to the coat protein. Five reactive lysine residues of CPMV coat protein provide sites to chemically conjugate to various compounds such as fluorescent dyes (Steinmetz, 2010). CPMV can be produced as empty virus like particles (eVLPs) Meshcheriakova et al. (2017). eVLPs are non-infectious and could be loaded with heterologous material, has increased the number of possible applications for CPMV-based particles.

\section{Biomedical Applications}

VLPs can act as strong vaccine candidates as they simulate the conformations of native viruses, utilizing their intrinsic immunogenicity without compromising their safety. VLPs evoke effectual immune responses as they are readily internalized by antigen presenting cells (APCs) and are ideal platforms for antigen processing and epitope presentation to immune cells. VLPs are composed of multiple copies of their capsid (coat) proteins, which when assembled appear as repetitive, multivalent molecular scaffolds. As a result, the multiple copies of coat protein can facilitate the multivalent 
presentation of antigens fused to their surface. Therefore, VLP vaccines afford superior immunogenicity compared to antigens in their soluble states. Additionally, plant viral VLPs and VNPs possess inherent adjuvant properties dispensing with the use of additional adjuvants to evoke strong immune responses (Table 1).

TMV VLPs have been utilized as epitope display systems in a variety of settings, with a first example being a polio vaccine by Haynes et al. (1986). Later, TMV was used as an epitope display vehicle for a malaria vaccine and others, including foot and mouth disease virus, human papillomavirus, norovirus, hepatitis B virus, influenza virus and human immunodeficiency virus (Turpen et al., 1995; Nooraei et al., 2021). Röder et al. (2017), were able to fuse a fluorescent protein to the C-terminus of the Tobacco mosaic virus (TMV) coat protein (CP) and also carried an N-terminal Foot-andmouth disease virus (FMDV) $2 \mathrm{~A}$ sequence. This enables the fusion protein to be cleaved from TMV.

Potato virus $\mathrm{X}$, in contrast to TMV, has a flexuous rod shape and requires its genomic RNA to self-assemble. PVX has been used extensively as an epitope display system for vaccine research. For example, Uhde-Holzem et al. (2016), reported genetically altered PVX which displayed Staphylococcus aureus protein A fragments on its surface, and proved to be easily functionalized with IgG to be used in biosensing plant viruses. VLPs of Papaya mosaic virus (PapMV), of the Potexvirius family, has been engineered for use as a seasonal flu trivalent vaccine (Carignan et al., 2015; Balke and Zeltins, 2020).

CPMV has also been developed as an autonomously replicating virus vector for the expression of either peptides or polypeptides in plants (Shahgolzari et al., 2020). Examples of CPMV used as an epitope presentation system include epitopes from the outer membrane (OM) protein $\mathrm{F}$ of Pseudomonas aeruginosa which were shown to protect mice against bacterial challenge, and an epitope expressing the 30 amino acid D2 domain of the fibronectin-binding protein (FnBP) from Staphylococcus aureus, which has been shown to be able to protect rats against endocarditis (Liu et al., 2005).

Recently, Albakri et al. (2020), explored how CPMV particles can activate human monocytes, dendritic cells (DCs) and macrophages. Monocytes, upon incubation with CPMV in vitro, released the chemokines CXCL10, MIP-1a and MIP$1 \beta$ into cell culture supernatants. Dendritic cells and monocytederived macrophages were also activated after incubation with CPMV, this activation is part of SYK signaling. Shukla et al. (2020) were able to demonstrate that CPMV outperformed many other types of VLPs to be a particularly strong immune stimulant.

The capacity for multifunctionality and multivalency makes plant nanoparticle platforms an ideal choice for theranostic applications (Beatty and Lewis 2019; Wang, 2019). Plant nanoparticles are capable of precise molecular imaging to achieve accurate cancer diagnosis and therapy (Ma et al., 2017). Delivery of imaging probes through nanostructures can improve the chances of early-stage cancer diagnosis through the use of multiple modalities to improve resolution, sensitivity, penetration, time, cost and on the top of all clinical relevance compared to the single imaging modalities (Key and Leary, 2014;
Shahgolzari et al., 2020). Drug conjugated nanoparticles administered intravenously target tumors, via the process of enhanced permeability and retention (EPR) effect depending on the type of tumor (Hansen et al., 2015).

Molecular imaging is an emerging biomedical field which facilitates the visualization, of biological mechanisms in vivo. Imaging technologies can include magnetic resonance imaging (MRI), computed tomography (CT), positron emission tomography (PET) and optical imaging, which enable the monitoring of molecular and cellular processes in normal and diseased conditions in living subjects. (Chung et al., 2020). Plant VLPs can be beneficial for molecular imaging technologies than synthetic nanoparticles, due to their short half-life in circulation and their lack of side effects (Steinmetz, 2010). Furthermore, plant VLPs can be developed to carry a wide array of contrast agents and fluorescent labels, as they can be modified with antibodies, peptides and aptamers to enable enhanced targeting to specific tissues and cells.

Magnetic resonance imaging (MRI) is a promising technology for the diagnosis of disease due to its high resolution and deep contrast, however, virus-based nanoparticles have been used to increase sensitivity (Pokorski et al., 2011). TMV can act as a carrier to deliver high payloads of MRI contrast imaging agents to diseased tissues (Michael A. Bruckman et al., 2013) and fluorescent dyes for biosensing and bioimaging (Wen et al., 2015). TMV's biological compatibility and multi-valency enables it to be a suitable carrier of in vivo imaging agents. For example, TMV rods have been conjugated to "BF3," a multiphoton absorbing fluorophore which permitted mouse brain imaging over an extended duration without crossing the blood-brain barrier (Niehl et al., 2016). A bimodal contrast agent has been prepared to target integrin $\alpha 2 \beta 1$ by loading the internal cavity of TMV nanoparticles with the complex of dysprosium (Dy3+) and the near-infrared fluorescence (NIRF) dye Cy7.5, as well as the externally conjugated with an Asp-GlyGlu-Ala (DGEA) peptide through a linker polyethylene glycol. This nanoparticle (Dy-Cy7.5-TMV-DGEA) was stable, displayed a low cytotoxicity and achieved a high resolution when targeted to PC-3 prostate cancer cells (Hu et al., 2017).

Tobacco Mosaic Virus has been used successfully for CD imaging, targeting atherosclerosis and thrombosis by using an NIR dye as well as a targeting peptide conjugated to TMV [96]. These targeted TMV particles were able to identify atherosclerotic lesions in ApoE-/- mice upon intravenous injection, showing that TMV can be used as a platform to detect at-risk lesions.

VNPs based on PVX have been conjugated to fluorescent reporters as well [135]. As mentioned earlier, the small fluorescent iLOV protein was expressed on the surface of PVX through genetic engineering and served as a fluorescent probe which could be of potential use in vivo imaging. Shukla et al. (2018), reportedly produced PVX VNPs that displayed mCherry or GFP on their N-termini in $N$. benthamiana plants. Significantly, fluorescent PVX could successfully be used for in vivo particle tracking in an HT-29 murine model, for in vitro imaging of HT-29 cells, and for tracing viral infection within plants. 
CPMV can also be engineered for intravital imaging (imaging living cells while they are in a multicellular organism) and improved permeability with a retention effect that improves tumor penetration (Beatty and Lewis, 2019). For in vivo imaging of tumors, CPMV-based VNPs have been successfully engineered to target specific tissues (Cho et al., 2014). These tumor targeting VNPs also provide biocompatible platforms for cancer therapy and intravital imaging (Beatty and Lewis, 2019).

Clinical treatment for cancer has been routinely addressed by chemotherapy (Hu et al., 2017). Regardless, the high recurrence of cancers as well as the fast clearance of anti-cancer drugs and non-targeted drug delivery necessitate the administration of maximum tolerable doses of drugs in cancer therapy, leading to increased toxicity and lower performance (Cano-Garrido et al., 2021). Therefore, drug delivery technologies that are highly targeted and promote active drug accumulation in tumors, in concert with reductions of dose requirements, could alleviate these concerns and augment treatment outcomes.

Plant virus VLPs have several attractive features that make them appropriate for targeted administration of therapeutic molecules. The anti-cancer drug doxorubicin (DOX), has been successfully delivered using VNPs and VLPs. TMV- and PVXderived VLPs and VNPs have been successfully used to deliver DOX (Finbloom et al., 2018). In this context, helical plant VNPs such as TMV and PVX, with high aspect ratios, have proven to be of great use in effective drug delivery. VNPs have shown great promise since their cargo-RNA functions as a ruler establishing the length of the virus particle and simple adsorption of DOX on their surface was shown to be effective for reducing tumor growth (Bruckman et al., 2013; Pitek et al., 2016).

TMV can be used as a carrier of peptides with therapeutic or targeting activity against various cancers. Trastuzumab is a cancer cell inhibiting monoclonal antibody that uses the binding sites of human epidermal growth factor receptor 2 (HER2). Trastuzumab-binding peptides (TBP) are immunogenic in nature and capable of initiating production of HER2-inhibiting antibodies to seize the growth of HER2-carrying cancer cells. TMV particles displaying TBP have been created to activate this immunogenicity (Tyulkina et al., 2011). Similarly, a delivery system reported as PhenPt-TMV, with anticancer drug phenanthriplatin loaded into a hollow TMV carrier, serves as an example of stimuli responsive system, as the release of drug is induced in the presence of acidic environment (Czapar et al., 2016). Along these lines, Tian et al. (2018) demonstrated that the Transacting Activation Transduction (TAT) peptide, conjugated to the external surface of TMV, augmented internalization along with an increased ability to escape endo/lysosomal compartments. Most of these VLPS exhibited uptake by dendritic cells and macrophages and proved to be highly immunogenic. Thus, therapeutic nucleic acids can be easily delivered to immune cells during cancer treatments.

Plant virus VNPs have been used for targeted administration of platinum-based drugs against cancer. This is important as $50 \%$ of chemotherapy treatments involve the use of these platinumderived drugs. TMV has been demonstrated to efficiently deliver Cisplatin (Franke et al., 2018) and Phenanthriplatin (Vernekar et al., 2018), both of which are platinum-based drugs. The drugs were loaded into the TMV VNP cavity using charge-driven interactions or by synthesizing stable covalent adducts. Such a TMV-based drug delivery system was proven to enable superior, targeted cytotoxicity as well as increased ease of uptake by cancer cells in in vitro systems using HepG2 and MCF-7 cancer cell lines (Liu et al., 2016).

Another anti-cancer drug, mitoxanthrone (MTO), is a topoisomerase II inhibitor and has been shown to be encapsulated by TMV (Lin and Steinmetz, 2018). VNPs exhibited superior tumor-reduction in mouse cancer models, while precluding severe cardiac outcomes that sometimes accompany direct delivery of MTO. Yet another antineoplastic and antimitotic drug, valine-citrulline monomethyl auristatin E (vcMMAE), was bound to the exterior of TMV VNPs which targeted non-Hodgkin's lymphoma. Internalization into endolysosomal compartments was reported (Kernan et al., 2017), most likely accompanied by the protease-mediated release of the drug. This system was efficient in terms of cytotoxicity towards the in vitro Karpas 299 non-Hodgkin's lymphoma cell line with an IC50 of $250 \mathrm{nM}$.

Helical plant virus nanoparticles (VLPs) have also been used as combination therapies to augment their immune efficacy. The PVX-DOX (doxorubicin) (Lee et al., 2017) combination was shown to be highly effective in stimulating cytokine/ chemokine levels while prolonging the survival of mice in melanoma models compared to that obtained through the administration of either PVX or DOX alone.

PVX displaying TNF related apoptosis inducing ligand (TRAIL) was used to promote the recruitment and activation of death receptors in vitro in HCC-38 primary ductal carcinoma, BT-549 ductal carcinoma and the MDA-MB-231 breast cancer cell lines (Le et al., 2017; Röder et al., 2018). In vivo mouse models also demonstrated that the PVX-TRAIL formulation potently inhibited tumor growth. PVX has also been used by displaying tumor necrosis factor (TNF)-related apoptosis inducing ligand (TRAIL) on the surface of VNPs. Multivalent display of TRAIL enabled increased recruitment and stimulation of death receptors expressed on cancer cell lines (Le et al., 2019). Similarly, this formulation was shown to successfully suppress tumor growth in mice breast cancer models.

An efficient and new drug delivery system has been reported for Non-Hodgkin's B cell lymphomas (NHL) based on PVX binding affinity towards malignant B cells. PVX loaded with monomethyl auristatin (MMAE) and administered to tissues harboring malignant B cells lead to inhibition of NHL growth in a mouse model (Shukla et al., 2020). Jobsri et al., 2015 reported a study in which PVX was conjugated to an idiotypic (Id) tumorassociated antigen (TAA) recombinant through a biotin/ streptavidin linker, that elicited a 7 times higher anti-Id IgG response compared to Id alone in a mouse B-cell lymphoma model. Cytokine profiling in these mice revealed that the induction of IFN- $\alpha$ and IL-12, also that TLR7 was essential for viral RNA recognition.

PVX nanoparticles are increasingly being used for immunotherapy of tumor microenvironments. The monoclonal antibodies of Herceptin or Trastuzumab can be loaded onto PVX nanofilaments, which successfully induced 
apoptosis in breast cancer cell lines (Esfandiari et al., 2016). PVX was used as an expression vector for a mutant form of the HPV16 E7 oncoprotein, by fusing it with lichenase. This elicited protection against tumor progression in mice by inducing a robust cytotoxic T-cell response (Demurtas et al., 2013).

Steinmetz et al. (2009), found that CPMV nanoparticles could bind to vimentin, a protein found on the surface of most cells. Vimentin is upregulated during tumor progression, making it an attractive target for cancer therapy. The fact that surface vimentin expression correlated with CPMV uptake in this study demonstrated the ability of CPMV to detect invasive cancer cells.

The tumor microenvironment poses a great challenge to immune clearance by virtue of being immunosuppressive and favoring immune escape of the tumors through the inhibition of anti-tumor T-cells (Chung et al., 2020). CPMV VLP nanoparticles were shown to decrease tumor growth in murine models of lung melanomas, ovarian, colon and breast tumors (Lizotte et al., 2016; Wang et al., 2019). Mechanistically, CPMV has been shown to reprogram the tumor microenvironment by recruitment of natural killer cells and neutrophils, while enabling the transition of M2 to M1 anti-tumor macrophages. This innate immune cell population subsequently combats the tumor leading to cell lysis. Most recently, Mao et al. (2021) have deduced which TLRs are responsible for these properties.

The icosahedral shape of CPMV capsid can be loaded with precise drug cargos to target tumor and cancer cells. CPMV VNPs have also been formulated as slow-release aggregates along with polyamidoamine generation 4 dendrimers (CPMV-G4) (Czapar et al., 2018), where they were shown to be effective in combating ovarian cancer in murine models, even when provided as a single dosage.

CPMV VLPs have been attached to TAAs (tumor associated antigens) using chemical conjugation, genetic fusion and enzyme-mediated ligation techniques. For example, the human epidermal growth factor receptor 2 (HER2) epitope, when conjugated to the icosahedral CPMV, was successfully delivered to the lymphatic system with enhanced uptake and activation of APCs that led to an augmented anti-HER2 immune response. The CPMV HER2 candidate vaccine slowed tumor progression and metastasis in mouse models, enhancing survival (Shukla et al., 2013). Importantly, CPMV-HER2 stimulated a predominantly Th1 immune response while Sesbania Mosaic Virus-HER2 and CCMV-HER2 induced mostly a Th2 response in mouse models, thus proving that the nature of the epitope carrier itself plays an essential role in regulating the Th1/ Th2 bias. This could be due to differences in epitope display on the surface of the VNPs as well as the capsid.

Cancer vaccines against carbohydrate antigens associated with tumors (TACAs) could be useful for diminishing tumor progression. Nevertheless, carbohydrates are weakly immunogenic and therefore, plant viruses used as carriers of these molecules could enhance the immune response to TACAs. CPMV-TACA conjugates targeting the Tn antigen (GalNAca-O-Ser/Thr) (Yin et al., 2012) were demonstrated to induce enhanced IgG titers, implicating heightened T-cell mediated immunity and antibody isotype switching in mouse models.
IgG binding to the Tn antigens were observed in experiments wherein mice sera were added to breast cancer cell lines.

The chemotherapeutic cyclophosphamide, when used in combination with CPMV VNPs, profoundly elicited tumor cell death, releasing extracellular TAAs and stimulating immune cell invasion in addition to augmenting TAA recognition and antigen presentation (Cai et al., 2019) in mouse tumor models. CPMV VNPs have also been administered in combination with CD47blocking antibodies (Wang and Steinmetz, 2019) which proved to have synergistic effects in combating tumor growth in murine ovarian tumor models, where it activated phagocytes, leading to stimulation of the adaptive immune response. Similar synergistic effects were observed when CPMV VNPs were used in combination with the anti-programmed cell death-1 checkpoint inhibitor (Lam et al., 2018). In addition to this, CPMV has been used successfully in promoting anti-tumor effects, when combined with radiation therapy. In this instance, CPMV was shown to enhance the recruitment of APCs, which in turn targeted the extracellular TAAs and phagocytosed them to induce a prolonged effectual immune response (Patel et al., 2018) in mice and canine models.

The CPMV-DOX conjugate was developed using eighty molecules of the chemotherapeutic drug doxorubicin (DOX), covalently bound to carboxylates at the external surface of the CPMV nanoparticle. This drug delivery vehicle was found to be more cytotoxic than free DOX when used in low concentration, however, CPMV-DOX cytotoxicity is time-delayed at higher concentrations (Aljabali et al., 2013). Cancer cells manage to resist immunotherapies owing to the immunosuppressive nature of tumors. CPMV nanoparticles have been reported as an in situ vaccine to stimulate an anti-tumor response and overcome local immunosuppression (Shukla et al., 2020). CPMV is also shown to be effective for ovarian cancer. The strategy for immunotherapy resulting antitumor efficacy is promising and involved the formation of aggregates of CPMV and polyamidoamine generation 4 dendrimers (CPMV-G4). Administration of CPMV-G4 effectively reduced ovarian cancer (Czapar et al., 2018). CPMV nanoparticles thus provide a therapeutic application for tumor targeting, intravital imaging and cancer therapy (Yildiz et al., 2013). Further exploration into the pharmacology of CPMV nanoparticles will further elucidate its roles in the immune response (Nkanga et al., 2021).

Patel et al. (2018), used CPMV nanoparticles in conjunction with radiotherapy to delay ovarian tumor growth in a mouse model. The treatment was able to result in an increase in tumor infiltrating lymphocytes (TILs), suggesting that this combined treatment could act as a future in situ tumor vaccine. Further studies by Wang and Steinmetz (2019) found that a protein known as CD47, that is widely expressed on tumor cells, prevents the action of $\mathrm{T}$ cells and phagocytic cells. The authors used a combination therapy of CD47-blocking antibodies and CPMV nanoparticles to act synergistically and elicit an anti-tumor immune response. The same research group also used low doses of cyclophosphamide (CPA) and CPMV nanoparticles as a combination therapy to successfully reduce mouse tumors in vivo (Wang and Steinmetz, 2019). 
TABLE 1 | Select Examples of Plant Virus Nanoparticles and their Medical Applications.

\begin{tabular}{|c|c|c|c|}
\hline Plant virus nanoparticle & Architecture & Use in cancer immunotherapy & Reference \\
\hline $\begin{array}{l}\text { Tobacco mosaic } \\
\text { virus (TMV) }\end{array}$ & Rod-shaped & $\begin{array}{l}\text { carrier for tumor associated carbohydrate antigens } \\
\text { Carrier of cancer drug phenanthriplatin in inner core of virus nanoparticle } \\
\text { In vivo imaging }\end{array}$ & $\begin{array}{l}\text { Yin et al. (2012) } \\
\text { Vernekar et al. (2018) } \\
\text { Chung et al. (2020) }\end{array}$ \\
\hline Potato virus $X$ (PVX) & Rod-shaped & $\begin{array}{l}\text { In vivo imaging } \\
\text { PVX-DOX (doxorubicin) combination therapy } \\
\text { displays tumor necrosis factor (TNF)-related apoptosis inducing ligand (TRAIL) on the } \\
\text { surface }\end{array}$ & $\begin{array}{l}\text { Shukla et al. (2018) } \\
\text { Lee et al. (2017) } \\
\text { Le et al. (2019) }\end{array}$ \\
\hline $\begin{array}{l}\text { Cowpea Mosaic virus } \\
\text { (CPMV) }\end{array}$ & $\begin{array}{l}\text { Icosahedral } \\
\text { shaped }\end{array}$ & $\begin{array}{l}\text { HER2 epitope displayed on virus surface } \\
\text { combination therapy of CD47-blocking antibodies and CPMV nanoparticles } \\
\text { slow-release aggregates comprised of polyamidoamine generation } 4 \text { dendrimers } \\
\text { (CPMV-G4) }\end{array}$ & $\begin{array}{l}\text { Shukla et al. (2013) } \\
\text { Wang and Steinmetz, } \\
\text { (2019) } \\
\text { Czapar et al. (2018) }\end{array}$ \\
\hline
\end{tabular}

\section{CONCLUSION AND FUTURE DIRECTIONS}

The use of plant virus nanoparticles (VNPs) as drug delivery carriers for the treatment of infectious and chronic diseases including cancer are advantageous when compared with naked drugs (Shoeb and Hefferon, 2019; Hefferon, 2018). The most promising nanoparticle systems have been adopted from naturally occurring plant viruses. Plant viruses are ideal for drug delivery as they are safe, non-infectious and nontoxic to humans (Beatty and Lewis, 2019). Cancer cells exhibit specific antigens on the surface of tumor cells which can be identified and targeted by plant-virus based nanoparticles, thus providing a clinical application of diagnosis and therapeutics for cancer. The most promising nano-scale systems have been adopted from naturally occurring plant viruses such as Tobacco mosaic virus (TMV), Cowpea mosaic virus (CPMV), Potato virus X (PVX) and many more. Currently, these new strategies are only applied in small scale production. As these approaches undergo further development, we will witness a spectrum of possible applications in the fields of medicine and biomedical engineering.

\section{REFERENCES}

Adams, M. J., Antoniw, J. F., Bar-Joseph, M., Brunt, A. A., Candresse, T., Foster, G. D., et al. (2004). Virology Division News: The New Plant Virus Family Flexiviridae and Assessment of Molecular Criteria for Species Demarcation. Arch. Virol. 149 (5), 1045-1060. doi:10.1007/s00705-004-0304-0

Albakri, M. M., Veliz, F. A., Fiering, S. N., Steinmetz, N. F., and Sieg, S. F. (2020). Endosomal Toll-like Receptors Play a Key Role in Activation of Primary Human Monocytes by Cowpea Mosaic Virus. Immunology 159 (2), 183-192. doi:10.1111/imm.13135

Aljabali, A. A. A., Shukla, S., Lomonossoff, G. P., Lomonossoff, N. F., and Steinmetz, D. J. (2013). CPMV-DOX Delivers. Mol. Pharmaceutics 10 (1), 3-10. doi:10.1021/mp3002057

Balke, I., and Zeltins, A. (2020). Recent Advances in the Use of Plant Virus-like Particles as Vaccines. Viruses 12 (3), 270. doi:10.3390/v12030270

Beatty, P. H., and Lewis, J. D. (2019). Cowpea Mosaic Virus Nanoparticles for Cancer Imaging and Therapy. Adv. Drug Deliv. Rev. 145, 130-144. doi:10.1016/ j.addr.2019.04.005

Boone, C. E., Wang, C., Lopez-Ramirez, M. A., Beiss, V., Shukla, S., Chariou, P. L., et al. (2020). Active Microneedle Administration of Plant Virus Nanoparticles
In the future, plant virus nanoparticles will need to be developed for high throughput manufacturing. This will require the dedication of facilities that can produce many grams of plant virus nanoparticles using tens of thousands of plants (McNulty et al., 2021). Today, manufacturing facilities have been generated for plant molecular farming, and adaptations could be tailored for nanoparticles (Fausther-Bovendo and Kobiger, 2021). The regulatory pathway will require more exploration to speed the process. More research regarding how plant virus nanoparticles act upon the immune system is underway and will be needed (Mao et al., 2021). Finally, the use of plant virus chimeras or semi-synthetic plant virus nanoparticles with novel properties must be explored, as well as novel modes of administration, such as microneedle patches (Boone et al., 2020).

\section{AUTHOR CONTRIBUTIONS}

All authors listed have made a substantial, direct, and intellectual contribution to the work and approved it for publication.

for Cancer In Situ Vaccination Improves Immunotherapeutic Efficacy. ACS Appl. Nano Mater. 3 (8), 8037-8051. doi:10.1021/acsanm.0c01506

Bruckman, M. A., Hern, S., Jiang, K., Flask, C. A., Yu, X., and Steinmetz, N. F. (2013). Tobacco Mosaic Virus Rods and Spheres as Supramolecular HighRelaxivity MRI Contrast Agents. J. Mater. Chem. B 1 (10), 1482-1490. doi:10.1039/С3ТB00461A

Brun, M. J., Gomez, E. J., and Suh, J. (2017). Stimulus-responsive Viral Vectors for Controlled Delivery of Therapeutics. J. Controlled Release 267, 80-89. doi:10.1016/j.jconrel.2017.08.021

Byun, D. J., Wolchok, J. D., Rosenberg, L. M., and Girotra, M. (2017). Cancer Immunotherapy - Immune Checkpoint Blockade and Associated Endocrinopathies. Nat. Rev. Endocrinol. 13 (4), 195-207. doi:10.1038/ nrendo.2016.205

Cai, H., Wang, C., Shukla, S., and Steinmetz, N. F. (2019). Cowpea Mosaic Virus Immunotherapy Combined with Cyclophosphamide Reduces Breast Cancer Tumor Burden and Inhibits Lung Metastasis. Adv. Sci. 6 (16), 1802281. doi:10.1002/advs.201802281

Cano-Garrido, O., Álamo, P., Sánchez-García, L., Falgàs, A., Sánchez-Chardi, A., Serna, N., et al. (2021). Biparatopic Protein Nanoparticles for the Precision Therapy of CXCR4+ Cancers. Cancers 13 (12), 2929. doi:10.3390/ cancers 13122929 
Carignan, D., Thérien, A., Rioux, G., Paquet, G., Gagné, M.-È. L., Bolduc, M., et al. (2015). Engineering of the PapMV vaccine platform with a shortened M2e peptide leads to an effective one dose influenza vaccine. Vaccine 33 (51), 7245-7253. doi:10.1016/j.vaccine.2015.10.123

Cho, C.-F., Shukla, S., Simpson, E. J., Steinmetz, N. F., Luyt, L. G., and Lewis, J. D. (2014). Molecular Targeted Viral Nanoparticles as Tools for Imaging Cancer. Methods Mol. Biol. (Clifton, N.J.) 1108, 211-230. doi:10.1007/978-1-62703751-8_16

Chung, Y. H., Cai, H., and Steinmetz, N. F. (2020). Viral Nanoparticles for Drug Delivery, Imaging, Immunotherapy, and Theranostic Applications. Adv. Drug Deliv. Rev. 156, 214-235. doi:10.1016/j.addr.2020.06.024

Czapar, A. E., Tiu, B. D. B., Veliz, F. A., Pokorski, J. K., and Steinmetz., N. F. (2018). Slow-Release Formulation of Cowpea Mosaic Virus for In Situ Vaccine Delivery to Treat Ovarian Cancer. Adv. Sci. 5 (5), 1700991. doi:10.1002/ advs.20170099110.1002/advs.201700991

Czapar, A. E., Zheng, Y.-R., Riddell, I. A., Shukla, S., Awuah, S. G., Lippard, S. J., et al. (2016). Tobacco Mosaic Virus Delivery of Phenanthriplatin for Cancer Therapy. ACS Nano 10 (4), 4119-4126. doi:10.1021/ acsnano. 5b 07360

Demurtas, O. C., Massa, S., Ferrante, P., Venuti, A., Franconi, R., Giuliano, G. A., et al. (2013). A Chlamydomonas-derived Human Papillomavirus 16 E7 vaccine induces specific tumor protection. PLoS One 23 (8), e61473. doi:10.1371/ journal.pone.0061473

Esfandiari, N., Arzanani, M. K., Soleimani, M., Kohi-Habibi, M., and Svendsen, W. E. (2016). A New Application of Plant Virus Nanoparticles as Drug Delivery in Breast Cancer. Tumor Biol. 37, 1229-1236. doi:10.1007/s13277-015-3867-3

Farokhzad, O. C. (2008). Nanotechnology for Drug Delivery: the Perfect Partnership. Expert Opin Drug Deliv. 5 (9), 927-929. doi:10.1517/ 17425247.5.9.927

Fausther-Bovendo, H., and Kobinger, G. (2021). Plant-made Vaccines and Therapeutics. Science 13, 740-741. doi:10.1126/science.abf5375

FDA Drug Products (2017). Including Biological Productsd, that Contain Nanomaterials-Guidance for Industry. Silver Spring, Maryland: FDA Drug Products.

Finbloom, J., Aanei, I., Bernard, J., Klass, S., Elledge, S., Han, K., et al. (2018). Evaluation of Three Morphologically Distinct Virus-like Particles as Nanocarriers for Convection-Enhanced Drug Delivery to Glioblastoma. Nanomaterials 8 (12), 1007. doi:10.3390/nano8121007

Franke, C. E., Czapar, A. E., Patel, R. B., and Steinmetz, N. F. (2018). Tobacco Mosaic Virus-Delivered Cisplatin Restores Efficacy in Platinum-Resistant Ovarian Cancer Cells. Mol. Pharmaceutics 15, 2922-2931. doi:10.1021/ acs.molpharmaceut.7b00466

Hansen, A. E., Petersen, A. L., Henriksen, J. R., Boerresen, B., Rasmussen, P., Elema, D. R., et al. (2015). Positron Emission Tomography Based Elucidation of the Enhanced Permeability and Retention Effect in Dogs with Cancer Using Copper64 Liposomes. ACS Nano 9 (7), 6985-6995. doi:10.1021/acsnano.5b01324

Haynes, J. R., Cunningham, J., von Seefried, A., Lennick, M., Garvin, R. T., and Shen, S.-H. (1986). Development of a Genetically-Engineered, Candidate Polio Vaccine Employing the Self-Assembling Properties of the Tobacco Mosaic Virus Coat Protein. Nat. Biotechnol. 4 (7), 637-641. doi:10.1038/ nbt0786-637

Hefferon, K. (2018). Repurposing Plant Virus Nanoparticles. Vaccines 6 (1), 11. doi:10.3390/vaccines6010011

Hu, H., Zhang, Y., Shukla, S., Gu, Y., Yu, X., and Steinmetz, N. F. (2017). Dysprosium-Modified Tobacco Mosaic Virus Nanoparticles for Ultra-highfield Magnetic Resonance and Near-Infrared Fluorescence Imaging of Prostate Cancer. ACS nano 11 (9), 9249-9258. doi:10.1021/acsnano.7b04472

Jobsri, J., Allen, A., Rajagopal, D., Shipton, M., Kanyuka, K., Lomonossoff, G. P., et al. (2015). Plant Virus Particles Carrying Tumour Antigen Activate TLR7 and Induce High Levels of Protective Antibody. PLoS One 10 (2), e0118096. doi:10.1371/journal.pone.0118096

Kernan, D. L., Wen, A. M., Pitek, A. S., and Steinmetz, N. F. (2017). Featured Article: Delivery of Chemotherapeutic vcMMAE Using Tobacco Mosaic Virus Nanoparticles. Exp. Biol. Med. (Maywood) 242, 1405-1411. doi:10.1177/ 1535370217719222

Kwon, S.-J., Park, M.-R., Kim, K.-W., Plante, C. A., Hemenway, C. L., and Kim, K.-H. (2005). cis-Acting Sequences Required for Coat Protein Binding and In Vitro Assembly of Potato Virus X. Virology 334 (1), 83-97. doi:10.1016/j.virol.2005.01.018
Lam, P., Lin, R. D., and Steinmetz, N. F. (2018). Delivery of Mitoxantrone Using a Plant Virus-Based Nanoparticle for the Treatment of Glioblastomas. J. Mater. Chem. B 6 (37), 5888-5895. doi:10.1039/C8TB01191E

Le, D. H. T., Commandeur, U., and Steinmetz, N. F. (2019). Presentation and Delivery of Tumor Necrosis Factor-Related Apoptosis-Inducing Ligand via Elongated Plant Viral Nanoparticle Enhances Antitumor Efficacy. ACS Nano 13, 2501-2510. doi:10.1021/acsnano.8b09462

Le, D. H. T., Lee, K. L., Shukla, S., Commandeur, U., and Steinmetz, N. F. (2017). Potato Virus X, a Filamentous Plant Viral Nanoparticle for Doxorubicin Delivery in Cancer Therapy. Nanoscale 9 (6), 2348-2357. doi:10.1039/ c6nr09099k

Leary, J., and Key, J. (2014). Nanoparticles for Multimodal In Vivo Imaging in Nanomedicine. Int. J. Nanomedicine 9, 711-726. doi:10.2147/ijn.s53717

Lee, K. L., Murray, A. A., Le, D. H. T., Sheen, M. R., Shukla, S., Commandeur, U., et al. (2017). Combination of Plant Virus Nanoparticle-Based In Situ Vaccination with Chemotherapy Potentiates Antitumor Response. Nano Lett. 17, 4019-4028. doi:10.1021/acs.nanolett.7b00107

Lin, R. D., and Steinmetz., N. F. (2018). Tobacco Mosaic Virus Delivery of Mitoxantrone for Cancer Therapy. Nanoscale 10 (34), 16307-16313. doi:10.1039/c8nr04142c

Liu, L., Cañizares, M. C., Monger, W., Perrin, Y., Tsakiris, E., Porta, C., et al. (2005). Cowpea Mosaic Virus-Based Systems for the Production of Antigens and Antibodies in Plants. Vaccine 23 (15), 1788-1792. doi:10.1016/ j.vaccine.2004.11.006

Liu, X., Wu, F., Tian, Y., Wu, M., Zhou, Q., Jiang, S., et al. (2016). Size Dependent Cellular Uptake of Rod-like Bionanoparticles with Different Aspect Ratios. Sci. Rep. 6 (1), 24567. doi:10.1038/srep24567

Lizotte, P. H., Wen, A. M., Sheen, M. R., Fields, J., Rojanasopondist, P., Steinmetz, N. F., et al. (2016). In Situ vaccination with Cowpea Mosaic Virus Nanoparticles Suppresses Metastatic Cancer. Nat. Nanotech 11 (3), 295-303. doi:10.1038/ nnano.2015.292

Ma, Y.-Y., Jin, K.-T., Wang, S.-B., Wang, H.-J., Tong, X.-M., Huang, D.-S., et al. (2017). Molecular Imaging of Cancer with Nanoparticle-Based Theranostic Probes. Contrast Media Mol. Imaging 2017, 1-11. doi:10.1155/2017/1026270

Mao, C., Beiss, V., Fields, J., Steinmetz, N. F., and Fiering, S. (2021). Cowpea Mosaic Virus Stimulates Antitumor Immunity through Recognition by Multiple MYD88-Dependent Toll-like Receptors. Biomaterials 275, 120914. doi:10.1016/j.biomaterials.2021.120914

Massumi, H., Poormohammadi, S., Pishyar, S., Maddahian, M., Heydarnejad, J., Hosseini-Pour, A., et al. (2014). Molecular Characterization and Field Survey of Iranian Potato Virus X Isolates. Virus Dis. 25 (3), 338-344. doi:10.1007/s13337014-0222-z

McNulty, M. J., Kelada, K., Paul, D., Nandi, S., and McDonald, K. (2021). Introducing Uncertainty Quantification to Techno-Economic Models of Manufacturing Field-Grown Plant-Made Products. doi:10.31224/osf.io/m8gvx

Meshcheriakova, Y., Durrant, A., Hesketh, E. L., Ranson, N. A., and Lomonossoff, G. P. (2017). Combining High-Resolution Cryo-Electron Microscopy and Mutagenesis to Develop Cowpea Mosaic Virus for Bionanotechnology. Biochem. Soc. Trans. 45 (6), 1263-1269. doi:10.1042/bst20160312

Moreira, A. F., Dias, D. R., and Correia, I. J. (2016). Stimuli-responsive Mesoporous Silica Nanoparticles for Cancer Therapy: A Review. Microporous Mesoporous Mater. 236, 141-157. doi:10.1016/j.micromeso.2016.08.038

Nemykh, M. A., Efimov, A. V., Novikov, V. K., Orlov, V. N., Arutyunyan, A. M. Drachev, V. A., et al. (2008). One More Probable Structural Transition in Potato Virus X Virions and a Revised Model of the Virus Coat Protein Structure. Virology 373 (1), 61-71. doi:10.1016/j.virol.2007.11.024

Niehl, A., Appaix, F., Boscá, S., van der Sanden, B., Nicoud, J.-F., Bolze, F., et al. (2016). Fluorescent Tobacco Mosaic Virus-Derived Bio-Nanoparticles for Intravital Two-Photon Imaging. Front. Plant Sci. 6, 1244. doi:10.3389/ fpls.2015.01244

Nkanga, C. I., and Steinmetz, N. F. (2021). The Pharmacology of Plant Virus Nanoparticles. Virology 556, 39-61. doi:10.1016/j.virol.2021.01.012

Nooraei, S., Bahrulolum, H., Hoseini, Z. S., Katalani, C., Hajizade, A., Easton, A. J., et al. (2021). Virus-like Particles: Preparation, Immunogenicity and Their Roles as Nanovaccines and Drug Nanocarriers. J. Nanobiotechnol 19 (1), 59. doi:10.1186/s12951-021-00806-7

Park, K. (2019). The Beginning of the End of the Nanomedicine Hype. J.Control. Release 305. doi:10.1016/j.jconrel.2019.05.044 
Park, M.-R., Kwon, S.-J., Choi, H.-S., Hemenway, C. L., and Kim, K.-H. (2008). Mutations that Alter a Repeated ACCA Element Located at the $5^{\prime}$ End of the Potato Virus X Genome Affect RNA Accumulation. Virology 378 (1), 133-141. doi:10.1016/j.virol.2008.05.004

Parker, L., Kendall, A., and Stubbs, G. (2002). Surface Features of Potato Virus X from Fiber Diffraction. Virology 300 (2), 291-295. doi:10.1006/viro.2002.1483

Patel, R., Czapar, A. E., Fiering, S., Oleinick, N. L., and Steinmetz, N. F. (2018). Radiation Therapy Combined with Cowpea Mosaic Virus Nanoparticle In Situ Vaccination Initiates Immune-Mediated Tumor Regression. ACS Omega 3 (4), 3702-3707. doi:10.1021/acsomega.8b00227

Paviolo, C., and Stoddart, P. (2017). Gold Nanoparticles for Modulating Neuronal Behavior. Nanomaterials 7, 92. doi:10.3390/nano7040092

Pitek, A. S., Jameson, S. A., Veliz, F. A., Shukla, S., and Steinmetz, N. F. (2016). Serum Albumin 'camouflage' of Plant Virus Based Nanoparticles Prevents Their Antibody Recognition and Enhances Pharmacokinetics. Biomaterials 89, 89-97. doi:10.1016/j.biomaterials.2016.02.032

Pokorski, J. K., Breitenkamp, K., Liepold, L. O., Qazi, S., and Finn, M. G. (2011). Functional Virus-Based Polymer-Protein Nanoparticles by Atom Transfer Radical Polymerization. J. Am. Chem. Soc. 133 (24), 9242-9245. doi:10.1021/ja203286n

Pokorski, J. K., and Steinmetz, N. F. (2011). The Art of Engineering Viral Nanoparticles. Mol. Pharmaceutics 8 (1), 29-43. doi:10.1021/mp100225y

Röder, J., Dickmeis, C., and Commandeur, U. (2019). Small, Smaller, Nano: New Applications for Potato Virus X in Nanotechnology. Front. Plant Sci. 10, 158. doi:10.3389/fpls.2019.00158

Röder, J., Dickmeis, C., Fischer, R., and Commandeur, U. (2018). Systemic Infection of Nicotiana Benthamianawith Potato Virus X Nanoparticles Presenting a Fluorescent iLOV Polypeptide Fused Directly to the Coat Protein. Biomed. Res. Int. 2018, e9328671. doi:10.1155/2018/9328671

Röder, J., Fischer, R., and Commandeur, U. (2017). Adoption of the 2A Ribosomal Skip Principle to Tobacco Mosaic Virus for Peptide Display. Front. Plant Sci. 8, 1125. doi:10.3389/fpls.2017.01125

Rong, J., Niu, Z., Lee, L. A., and Wang, Q. (2011). Self-assembly of Viral Particles. Curr. Opin. Colloid Interf. Sci. 16 (6): 441-450. doi:10.1016/j.cocis.2011.09.001

Rybicki, E. P. (2020). Plant Molecular Farming of Virus-like Nanoparticles as Vaccines and Reagents. WIREs Nanomed Nanobiotechnol 12 (2), e1587. doi:10.1002/wnan.1587

Sainsbury, F., Cañizares, M. C., and Lomonossoff, G. P. (2010). Cowpea mosaicVirus: The Plant Virus-Based Biotechnology Workhorse. Annu. Rev. Phytopathol. 48, 437-455. doi:10.1146/annurev-phyto-073009-114242

Shahgolzari, M., Pazhouhandeh, M., Milani, M., Yari Khosroushahi, A., and Fiering, S. (2020). Plant Viral Nanoparticles for Packaging and In Vivo Delivery of Bioactive Cargos. Wiley Interdiscip Rev Nanomed Nanobiotechnol. 12 (5), e1629. doi:10.1002/wnan.1629

Shahgolzari, M., Dianat-Moghadam, H., and Fiering, S. (2021). Multifunctional Plant Virus Nanoparticles in the Next Generation of Cancer Immunotherapies. Semin. Cancer Biol. 2021, S1044. doi:10.1016/j.semcancer.2021.07.018

Shoeb, E., and Hefferon, K. (2019). Future of Cancer Immunotherapy Using Plant Virus-Based Nanoparticles. Future Sci. OA 5 (7), FSO401. doi:10.2144/fsoa2019-0001

Shukla, S., Ablack, A. L., Wen, A. M., Lee, K. L., Lewis, J. D., and Steinmetz, N. F. (2013). Increased Tumor Homing and Tissue Penetration of the Filamentous Plant Viral Nanoparticle Potato Virus X. Mol. Pharmaceutics 10 (1), 33-42. doi: $10.1021 / \mathrm{mp} 300240 \mathrm{~m}$

Shukla, S., Dickmeis, C., Fischer, R., Commandeur, U., and Steinmetz, N. F. (2018). In Planta Production of Fluorescent Filamentous Plant Virus-Based Nanoparticles. Methods Mol. Biol. 1776, 61-84. doi:10.1007/978-1-4939-7808-3_5

Shukla, S., Roe, A. J., Liu, R., Veliz, F. A., Commandeur, U., Wald, D. N., et al. (2020). Affinity of Plant Viral Nanoparticle Potato Virus X (PVX) towards Malignant B Cells Enables Cancer Drug Delivery. Biomater. Sci. 8, 3935-3943. doi:10.1039/D0BM00683A

Shukla, S., Wang, C., Beiss, V., Cai, H., Washington, T., Murray, A. A., et al. (2020). The Unique Potency of Cowpea Mosaic Virus (CPMV) In Situ Cancer Vaccine. Biomater. Sci. 8 (19), 5489-5503. doi:10.1039/D0BM01219J10.1039/D0BM01219J

Singh, P., Prasuhn, D., Yeh, R. M., Destito, G., Rae, C. S., Osborn, K., et al. (2007). Bio-distribution, Toxicity and Pathology of Cowpea Mosaic Virus Nanoparticles In Vivo. J. Controlled Release 120, 41-50. doi:10.1016/ j.jconrel.2007.04.003
Steinmetz, N. F., and Evans, D. J. (2007). Utilisation of Plant Viruses in Bionanotechnology. Org. Biomol. Chem. 5 (18), 2891-2902. doi:10.1039/ b708175h

Steinmetz, N. F., and Manchester., M. (2009). PEGylated Viral Nanoparticles for Biomedicine: the Impact of PEG Chain Length on VNP Cell Interactions In Vitro and Ex Vivo. Biomacromolecules 10 (4), 784-792. doi:10.1021/bm8012742

Steinmetz, N. F., Shah, S. N., Barclay, J. E., Rallapalli, G., Lomonossoff, G. P., and Evans, D. J. (2009). Virus-templated Silica Nanoparticles. Small 5 (7), 813-816. doi:10.1002/smll.200801348

Steinmetz, N. F. (2010). Viral Nanoparticles as Platforms for Next-Generation Therapeutics and Imaging Devices. Nanomedicine: Nanotechnology, Biol. Med. 6 (5), 634-641. doi:10.1016/j.nano.2010.04.005

Tian, Y., Zhou, M., Shi, H., Gao, S., Xie, G., Zhu, M., et al. (2018). Integration of Cell-Penetrating Peptides with Rod-like Bionanoparticles: Virus-Inspired Gene-Silencing Technology. Nano Lett. 18, 5453-5460. doi:10.1021/ acs.nanolett.8b01805

Turpen, T. H., Reinl, S. J., Charoenvit, Y., Hoffman, S. L., Fallarme, V., and Grill, L. K. (1995). Malaria Epitopes Expressed on the Surface of Recombinant Tobacco Mosaic Virus. Nat. Biotechnol. 13 (1), 53-57. doi:10.1038/nbt0195-53

Tyulkina, L. G., Skurat, E. V., Frolova, O. Y., Komarova, T. V., Karger, E. M., and Atabekov, I. G. (2011). New Viral Vector for Superproduction of Epitopes of Vaccine Proteins in Plants. Acta naturae 3 (4), 73. doi:10.32607/207582512011-3-4-73-82

Uhde-Holzem, K., McBurney, M., Tiu, B. D., Advincula, R. C., Fischer, R., Commandeur, U., et al. (2016). Production of Immunoabsorbent Nanoparticles by Displaying Single-Domain Protein A on Potato Virus X. Macromol. Biosci. 16, 231-241. doi:10.1002/mabi.201500280

Vernekar, A. A., Berger, G., Czapar, A. E., Veliz, F. A., Wang, D. I., Steinmetz, N. F., et al. (2018). Speciation of Phenanthriplatin and its Analogs in the Core of Tobacco Mosaic Virus. J. Am. Chem. Soc. 140, 4279-4287. doi:10.1021/ jacs.7b12697

Wang, C., and Steinmetz, N. F. (2019). CD47 Blockade and Cowpea Mosaic Virus Nanoparticle In Situ Vaccination Triggers Phagocytosis and Tumor Killing. Adv. Healthc. Mater. 8 (8), e1801288. doi:10.1002/adhm.201801288

Wang, C., Beiss, V., and Steinmetz, N. F. (2019). Cowpea Mosaic Virus Nanoparticles and Empty Virus-like Particles Show Distinct but Overlapping Immunostimulatory Properties. J. Virol. 93 (21), e00129-19. doi:10.1128/JVI.00129-19

Wen, A. M., Infusino, M., De Luca, A., Kernan, D. L., Czapar, A. E., Strangi, G., et al. (2015b). Interface of Physics and Biology: Engineering Virus-Based Nanoparticles for Biophotonics. Bioconjug. Chem. 26 (1), 51-62. doi:10.1021/bc500524f

Wen, A. M., Wang, Y., Jiang, K., Hsu, G. C., Gao, H., Lee, K. L., et al. (2015a). Shaping Bio-Inspired Nanotechnologies to Target Thrombosis for Dual Optical-Magnetic Resonance Imaging. J. Mater. Chem. B 3 (29), 6037-6045. doi:10.1039/c5tb00879d

Wen, J., Xu, Y., Li, H., Lu, A., and Sun, S. (2015b). Recent Applications of Carbon Nanomaterials in Fluorescence Biosensing and Bioimaging. Chem. Commun. 51 (57), 11346-11358. doi:10.1039/C5CC02887F

Yildiz, I., Lee, K. L., Chen, K., Shukla, S., and Steinmetz, N. F. (2013). Infusion of imaging and therapeutic molecules into the plant virus-based carrier cowpea mosaic virus: cargo-loading and delivery. J Control Release 172 (2), 568-78. doi:10.1016/j.jconrel.2013.04.023

Yin, Z., Nguyen, H. G., Chowdhury, S., Bentley, P., Bruckman, M. A., Miermont, A., et al. (2012). Tobacco Mosaic Virus as a New Carrier for Tumor Associated Carbohydrate Antigens. Bioconjug. Chem. 23, 1694-1703. doi:10.1021/ bc300244a

Young, M., Debbie, W., Uchida, M., and Douglas, T. (2008). Plant Viruses as Biotemplates for Materials and Their Use in Nanotechnology. Annu. Rev. Phytopathol. 46, 361-384. doi:10.1146/annurev.phyto.032508.131939

Zeng, Q., Wen, H., Wen, Q., Chen, X., Wang, Y., Xuan, W., et al. (2013). Cucumber Mosaic Virus as Drug Delivery Vehicle for Doxorubicin. Biomaterials 34 (19), 4632-4642. doi:10.1016/j.biomaterials.2013.03.017

Zhang, L., Gu, F., Chan, J., Wang, A., Langer, R., and Farokhzad, O. (2008). Nanoparticles in Medicine: Therapeutic Applications and Developments. Clin. Pharmacol. Ther. 83, 761-769. doi:10.1038/sj.clpt.6100400

Zhou, J. C., Soto, C. M., Chen, M.-S., Bruckman, M. A., Moore, M. H., Barry, E., et al. (2012). Biotemplating Rod-like Viruses for the Synthesis of Copper 
Nanorods and Nanowires. J. Nanobiotechnology 10, 18. doi:10.1186/1477-3155$10-18$

Conflict of Interest: The authors declare that the research was conducted in the absence of any commercial or financial relationships that could be construed as a potential conflict of interest.

Publisher's Note: All claims expressed in this article are solely those of the authors and do not necessarily represent those of their affiliated organizations, or those of the publisher, the editors and the reviewers. Any product that may be evaluated in this article, or claim that may be made by its manufacturer, is not guaranteed or endorsed by the publisher.

Copyright $\odot 2021$ Venkataraman, Apka, Shoeb, Badar and Hefferon. This is an open-access article distributed under the terms of the Creative Commons Attribution License (CC BY). The use, distribution or reproduction in other forums is permitted, provided the original author(s) and the copyright owner(s) are credited and that the original publication in this journal is cited, in accordance with accepted academic practice. No use, distribution or reproduction is permitted which does not comply with these terms. 